\title{
Human thioredoxin-1 attenuates the rate of lipopolysaccharide-induced preterm delivery in mice in association with its anti-inflammatory effect
}

\author{
Fumihiko Namba', Mikiko Kobayashi-Miura², Taro Goda ${ }^{3}$, Yukiko Nakura', Fumiko Nishiumi', Aoi Son², Akio Kubota4, \\ Junji Yodoi ${ }^{2}$ and Itaru Yanagihara'
}

\begin{abstract}
BACKGROUND: Maternal intrauterine infection/inflammation represents the major etiology of preterm delivery and the leading cause of neonatal mortality and morbidity. The aim of this study was to investigate the anti-inflammatory properties of thioredoxin-1 in vivo and its potential ability to attenuate the rate of inflammation-induced preterm delivery.

METHODS: Two intraperitoneal injections of lipopolysaccharide from Escherichia coli were administered in pregnant mice on gestational day 15, with a 3-h interval between the injections. From either $1 \mathrm{~h}$ before or $1 \mathrm{~h}$ after the first lipopolysaccharide injection, mice received three intravenous injections of either recombinant human thioredoxin-1, ovalbumin, or vehicle, with a 3-h interval between injections.
\end{abstract}

RESULTS: Intraperitoneal injection of lipopolysaccharide induced a rise of tumor necrosis factor- $\alpha$, interferon- $\gamma$, monocyte chemotactic protein 1, and interleukin-6 in maternal serum levels and provoked preterm delivery. Recombinant human thoredoxin-1 prevented the rise in these proinflammatory cytokine levels. After the inflammatory challenge, placentas exhibited severe maternal vascular dilatation and congestion and a marked decidual neutrophil activation. These placental pathological findings were ameliorated by recombinant human thioredoxin-1, and the rate of inflammation-induced preterm delivery was attenuated.

CONCLUSION: Thioredoxin-1 may thus represent a novel effective treatment to delay inflammation-induced preterm delivery.

T he estimated number of preterm births (defined as birth before gestational week 37) worldwide was 15 million in 2010 (11.1\%) (1). Preterm birth is the most frequent cause of infant mortality and leads to one million deaths per year. Premature infants face increased risk of neonatal complications, such as intraventricular hemorrhage, bronchopulmonary dysplasia, retinopathy, and necrotizing enterocolitis as well as increased risk of adult-onset obesity, diabetes, and hypertension $(2,3)$.
Intrauterine infection or inflammation caused by various bacteria, including Escherichia coli, Gardnerella vaginalis, anaerobic bacteria, and genital mycoplasmas, can lead to preterm birth $(4,5)$. Animal models of bacterial componentinduced preterm birth have been established $(6,7)$.

Thioredoxin-1 (TRX), originally cloned as a soluble factor called adult T-cell leukemia-derived factor (8), is one of the most important molecules controlling the redox regulation system and contains a redox-active disulfide/dithiol within the conserved active site sequence Cys ${ }^{32}$-Gly-Pro-Cys ${ }^{35}$ (ref. (9)). TRX has a pivotal role in scavenging reactive oxygen species (ROS) with peroxiredoxins, and thus, it prevents apoptosis of various cells (10). Moreover, circulating TRX inhibits neutrophil infiltration into the sites of inflammation by blocking the adhesion of lipopolysaccharide (LPS)-stimulated neutrophils on endothelial cells (11). Overexpression of human TRX in transgenic mice induced resistance to harmful conditions, including thioacetamide- or LPS-induced acute hepatitis (12), and proinflammatory cytokine- or bleomycin-induced lung injury (13). These findings suggest that TRX has protective effects on various diseases, possibly due to its antioxidative and anti-inflammatory actions. However, little is known about the role of TRX in intrauterine inflammation.

In the present study, we investigated the ability of TRX to attenuate the rate of preterm delivery in vivo in a murine model of intrauterine inflammation. We found that intravenous administration of recombinant human thioredoxin-1 (rhTRX) reduced the rate of inflammation-induced preterm delivery by suppressing maternal proinflammatory cytokine production and placental pathological changes, including vascular dilatation and congestion, and decidual neutrophil infiltration.

\section{RESULTS \\ Prophylactic Effect of TRX on Inflammation-Induced Preterm Delivery}

First, to investigate whether exogenous TRX has prophylactic efficacy in preterm delivery, we intravenously administered rhTRX to LPS-treated pregnant mice using the

\footnotetext{
'Department of Developmental Medicine, Osaka Medical Center and Research Institute for Maternal and Child Health, Osaka, Japan; ${ }^{2}$ Department of Biological Responses, Infection and Prevention, Institute for Virus Research, Kyoto University, Kyoto, Japan; ${ }^{3}$ Department of Surgery, Izumiotsu Municipal Hospital, Osaka, Japan; ${ }^{4}$ Second Department of Surgery, Wakayama Medical University, Wakayama, Japan. Correspondence: Itaru Yanagihara (itaruy@mch.pref.osaka.jp)

Received 1 October 2015; accepted 3 March 2016; advance online publication 25 May 2016. doi:10.1038/pr.2016.100
} 


\section{Articles | Nambaetal.}

a

(prophylactic protocol)

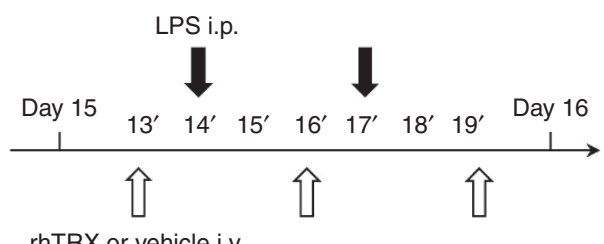

rhTRX or vehicle i.v.

b

(therapeutic protocol)

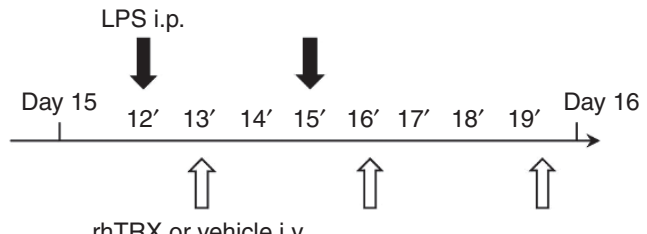

rhTRX or vehicle i.v.

Figure 1. Schematic summary of experimental protocol for lipopolysaccharide (LPS) induced preterm delivery in mice. (a) Prophylactic protocol. The 15-d gestation $\mathrm{C} 3 \mathrm{H} / \mathrm{HeN}$ mice were injected i.p. with $200 \mu \mathrm{g} / \mathrm{kg}$ of LPS of Escherichia coli (serotype 055: B5) dissolved in $10 \mathrm{ml} / \mathrm{kg}$ of sterile saline twice with a 3 -h interval (at 0 and $3 \mathrm{~h}$ ). From $1 \mathrm{~h}$ before first LPS injection, mice received $10 \mathrm{ml} / \mathrm{kg}$ of phosphate-buffered saline containing either $\mathrm{rhTRX} 1(2 \mathrm{mg} / \mathrm{kg})$, OVA $(2 \mathrm{mg} / \mathrm{kg})$, or vehicle three times with a 3-h interval (at -1, 2, and 5 h). (b) Therapeutic protocol. After $1 \mathrm{~h}$ after first LPS injection, mice received $10 \mathrm{ml} / \mathrm{kg}$ of phosphate-buffered saline containing either rhTRX1 ( $2 \mathrm{mg} / \mathrm{kg})$ or vehicle three times with a 3-h interval (at 1,4 , and $7 \mathrm{~h}$ ).

Table 1. Effect of prophylactic TRX on a LPS-induced preterm delivery mouse model

\begin{tabular}{lcccc}
\hline & & \multicolumn{2}{c}{ Incidence $(n)$} & \\
\cline { 3 - 4 } Group & Mice $(n)$ & $\begin{array}{l}\text { Preterm } \\
\text { delivery }\end{array}$ & Term delivery & $\begin{array}{c}\text { Rate of preterm } \\
\text { delivery }(\%)\end{array}$ \\
\hline Control & 14 & 0 & 14 & 0 \\
LPS & 16 & 15 & 1 & $94^{* *}$ \\
LPS-PBS & 10 & 10 & 0 & $100^{* *}$ \\
LPS-OVA & 9 & 8 & 1 & $89^{* *}$ \\
LPS-TRX & 10 & 4 & 6 & $40^{*,+\dagger}$ \\
\hline
\end{tabular}

Prophylactic protocol, from $1 \mathrm{~h}$ before the first LPS injection, mice received three i.v. injections of PBS containing either TRX, OVA, or vehicle, with a 3-h interval between injections (at $-1,2$, and 5 h) (Figure 1a).

Fisher's exact test was applied for comparison of preterm delivery rate in each group. ${ }^{*} P<0.05,{ }^{* *} P<0.01$ vs. control, ${ }^{+\dagger} P<0.01$ vs. LPS

LPS, lipopolysaccharide; OVA, ovalbumin; PBS, phosphate-buffered saline; TRX, Thioredoxin-1.

protocols described (Figure 1a). In the prophylactic protocol, intraperitoneal (i.p.) injection of LPS provoked preterm delivery in 15 of 16 treated animals (94\%). No maternal mortality was observed. The rates of preterm delivery in the phosphate-buffered saline (PBS)-treated group and ovalbumin (OVA)-treated group were 100\% (10/10) and 89\% (8/9), respectively. Treatment with rhTRX $1 \mathrm{~h}$ before the LPS injection significantly reduced the rate of preterm delivery ( $40 \mathrm{vs.}$ $94 \% ; P<0.01$ ), whereas the rates of preterm delivery did not change after treatment with PBS or OVA (Table 1).
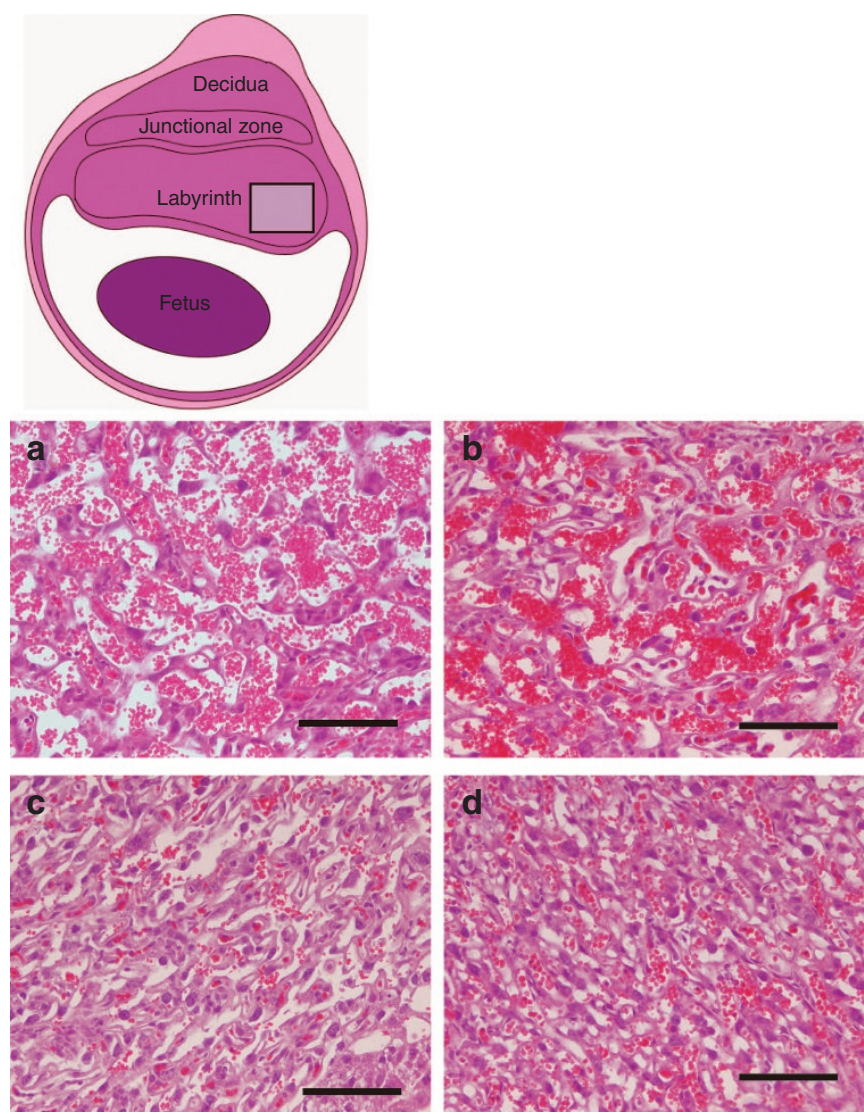

Figure 2. Inhibition of blood congestion and vascular dilatation in the placenta by prophylactic thioredoxin-1 (TRX). Diagram representing uteroplacental units on 15th gestational day. Square, regions photographed in the labyrinth. Hematoxylin and eosin stain of (a) lipopolysaccharide (LPS), (b) LPS-PBS, (c) LPS-TRX, and (d) nontreated dams, $6 \mathrm{~h}$ after first LPS injection. Representative sections obtained from five different animals per group are shown. Scale bar, $100 \mu \mathrm{m}$. PBS, phosphate-buffered saline.

\section{Inhibition of Inflammation-Induced Histological Changes in the Placenta by TRX}

To investigate whether prophylactic administration of rhTRX ameliorates placental inflammation in LPS-induced preterm delivery, sections of uteroplacental tissue from the four groups of animals (untreated, LPS-treated, LPS-treated with either PBS or rhTRX) were mounted. Histological aspects were assessed using hematoxylin and eosin stain, and the extent of inflammatory cell infiltration was assessed using neutrophil immunohistochemistry. Histological analysis of placental sections from LPS-treated mice revealed maternal blood congestion and vascular dilatation in the placental labyrinth (Figure 2) and neutrophil activation which was demonstrated by deformed or adhered neutrophils at around decidual vessels from maternal blood sinuses (Figure 3a). In contrast, TRXtreated animals before LPS injection exhibited reduced maternal blood congestion and vascular dilatation in the labyrinth (Figure 2d). Furthermore, the number of deformed or adhered activated neutrophil in the decidua was decreased in the TRXtreated group (LPS-TRX) compared with the LPS-treated 

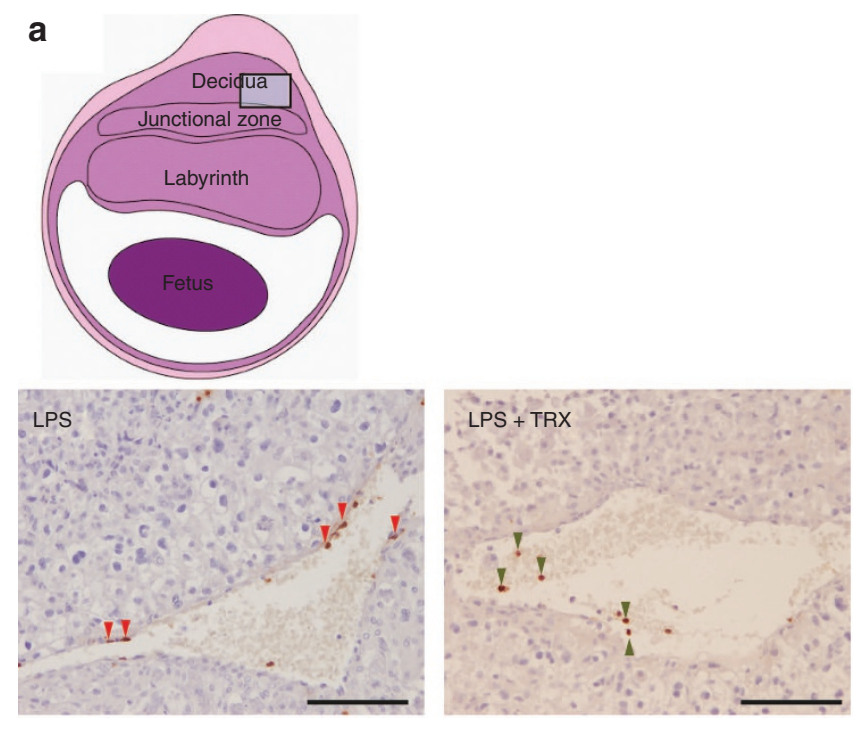

b

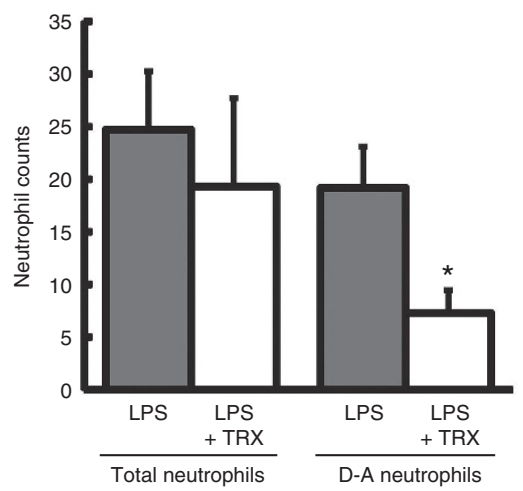

Figure 3. Inhibition of neutrophil activation from maternal blood sinus in the placenta by prophylactic thioredoxin-1 (TRX). (a) Diagram representing uteroplacental units on 15th gestational day. Square, regions photographed in the decidua. Neutrophil immunostaining in lipopolysaccharide (LPS) and LPS-TRX dams, $6 \mathrm{~h}$ after first LPS injection. Red arrow heads, the deformed or adhered activated neutrophils at around decidual vessels of placenta. Dark Green arrow heads, normal shaped neutrophils. (b) Number of total and activated neutrophils in the decidua counted in four mice placentas from each group (LPS and LPS-TRX). At least four microscopic photographs were taken in each placenta. ${ }^{*} P<0.05$ : significantly different from LPS-treated group. D-A (deformed or activatied) neutrophils. Scale bar, $100 \mu \mathrm{m}$.

group, although no changes in total neutrophil counts in the decidua were observed between these two groups (Figure $3 \mathbf{b}$ ).

\section{Decrease in Serum Levels of Inflammation-Induced Cytokines by TRX}

To determine whether the onset of preterm labor in LPStreated animals was concomitant to a maternal systemic or intrauterine inflammation and whether this phenomenon was prevented in the presence of prophylactic TRX, the levels of cytokines (interleukin (IL)-12, tumor necrosis factor- $\alpha$ (TNF- $\alpha$ ), interferon- $\gamma$ (IFN- $\gamma$ ), monocyte chemotactic protein 1 (MCP-1), IL-10, and IL-6) in maternal sera were measured. As depicted in Figure 4, i.p. injection of LPS induced a significant increase in TNF- $\alpha$, IFN- $\gamma$, MCP-1, and IL- 6 levels in the maternal serum compared with untreated control animals $6 \mathrm{~h}$ after the first LPS injection, demonstrating that a systemic inflammation was mounted against LPS in these animals. LPS i.p. injection did not significantly affect the levels of IL-12 and IL-10 in the maternal serum. The injection of TRX significantly diminished the increase in TNF- $\alpha$, IFN- $\gamma$, MCP1 , and IL-6 levels in maternal serum of LPS-treated animals $(P<0.01)$ while not significantly affecting IL-12 and IL-10 levels (Figure 4).

No Change in Serum Levels of Macrophage Migration Inhibitory Factor After Prophylactic TRX Administration During Intrauterine Inflammation

Serum macrophage migration inhibitory factor (MIF) levels were significantly higher in pregnant mice than in nonpregnant mice $(P<0.01)$ and in the LPS-induced preterm delivery mouse model than in untreated normal pregnant mice $(P<0.05)$. High serum MIF levels were observed in all three
LPS-treated groups (LPS, LPS-PBS, and LPS-TRX), without significant difference between the three groups (Figure 5).

\section{Therapeutic Effect of TRX on Inflammation-Induced Preterm Delivery}

To investigate whether exogenous TRX has therapeutic efficacy in preterm delivery, rhTRX was intravenously administered to LPS-treated pregnant mice using the therapeutic protocol (Figure 1b). I.p. injection of LPS provoked preterm delivery in 15 of 22 treated animals (68\%). The rates of preterm delivery in the PBS-treated group and the TRX-treated group were $68 \%(23 / 34)$ and $41 \%(14 / 34)$, respectively. The rate of preterm delivery was significantly lower in the TRX-treated group than in the PBS-treated group in the therapeutic protocol (41 vs. $68 \% ; P<0.05)$ (Table 2).

\section{Inhibitory Effect of TRX on LPS-Induced ROS Production in the Mouse Macrophages}

To elucidate the inhibitory effect of TRX on ROS production, ROS in a primary culture of mouse peritoneal macrophages were measured using methods previously reported (14). LPS $(2 \mu \mathrm{g} / \mathrm{ml})$ induced ROS production in mice peritoneal macrophages while TRX $(20 \mu \mathrm{g} / \mathrm{ml})$ significantly decreased the ROS derived fluorescence after LPS treatment (Figure 6a,b). TRX revealed LPS-induced ROS-scavenging properties in vitro.

\section{DISCUSSION}

In the present study, we report for the first time that TRX blocks systemic and placental inflammation in vivo and attenuates the rate of preterm delivery in a mouse model. Preterm delivery is a leading cause of neonatal mortality and morbidity. To date, several tocolytic agents, such as the $\beta$-adrenergic 


\section{Articles $\mid$ Nambaet al.}

a

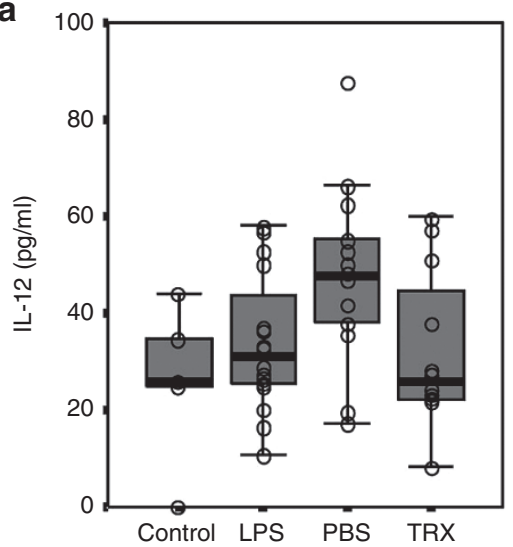

d

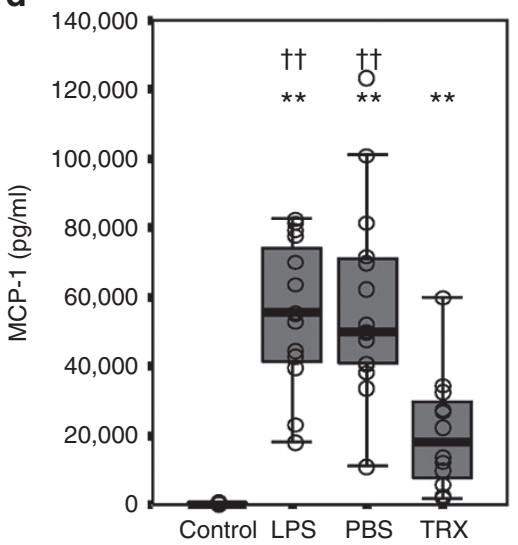

b

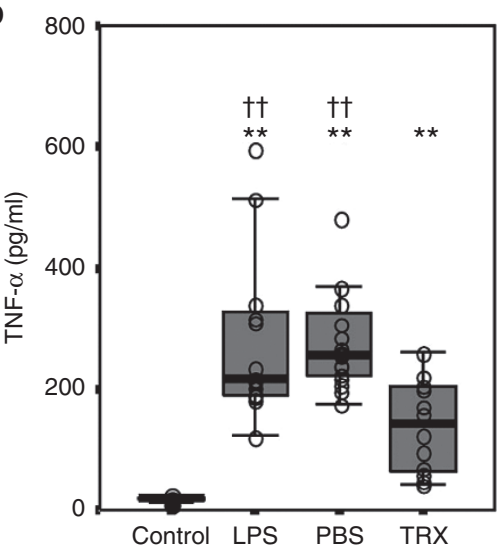

e

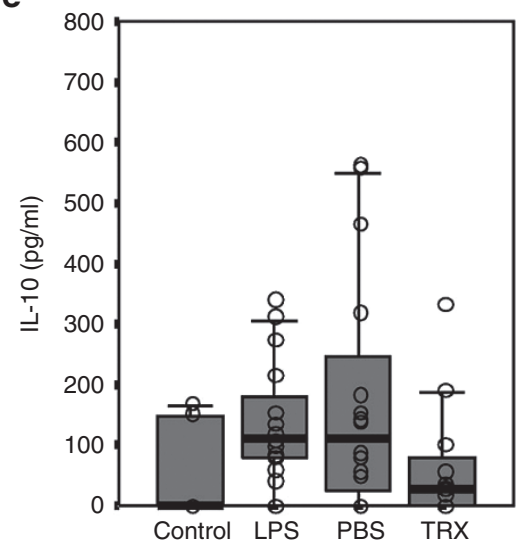

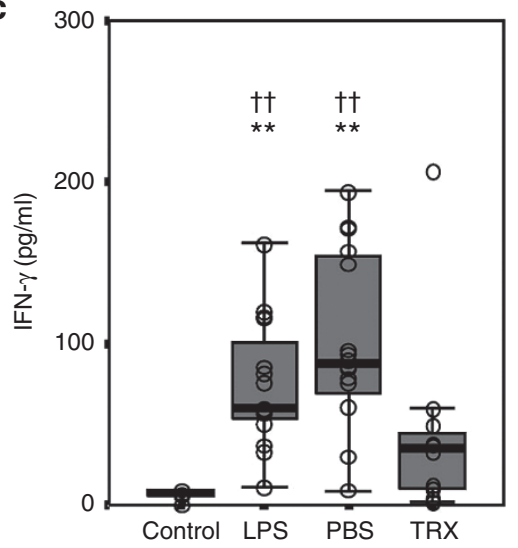

f

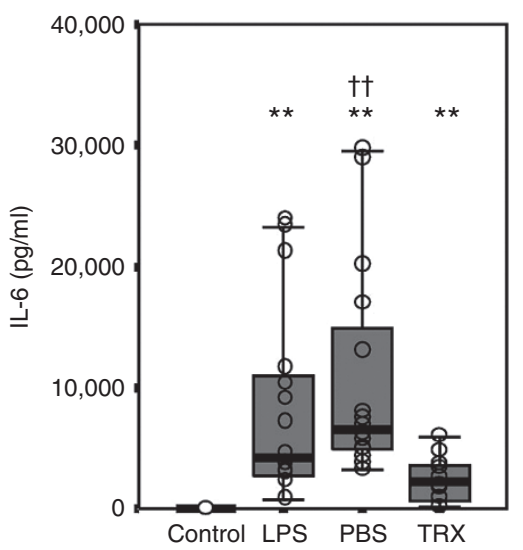

Figure 4. Inhibition of inflammation-induced cytokine production in maternal serum by prophylactic thioredoxin-1 (TRX). Concentration of (a) IL-12, (b) TNF- $\alpha$, (c) IFN- $\gamma$, (d) MCP-1, (e) IL-10, and (f) IL-6 were measured by Cytometric Bead Array in maternal serum of nontreated (control), lipopolysaccharide (LPS) (LPS), LPS-PBS (PBS), and LPS-TRX (TRX) dams, $6 \mathrm{~h}$ after first LPS injection. Box-and-whisker representations indicate the median and the overall range of distribution of the measurements, the bars indicating the smallest and greatest value except for outliers. ${ }^{*} P<0.01$ : significantly different from nontreated group. ${ }^{\dagger+} P<0.01$ : significantly different from TRX-treated group. PBS, phosphate-buffered saline.

agonist ritodrine and magnesium sulfate, have been used to prolong pregnancy (15). An analysis of 36 randomized controlled trials demonstrated that prenatal administration of progesterone prevented preterm birth (16). Nonetheless, the incidence of preterm birth continues to rise $(17,18)$.

Evidence from animal models of preterm birth also strongly suggest that several molecules, including phosphodiesterase- 4 inhibitor, cyclopentenone prostaglandin, anti-TNF- $\alpha$, matrix metalloproteinase inhibitor, and folic acid, may be useful to treat inflammation-induced preterm labor (19-23). In addition to these agents, antioxidants are believed to represent a promising treatment option, not only for preterm birth $(7,14,24)$ but also for neonatal disorders, bronchopulmonary dysplasia, retinopathy of prematurity, necrotizing enterocolitis, and periventricular leukomalacia (25). We showed that TRX is one of the potential agents for intrauterine infection/inflammation by its antioxidant and anti-inflammatory properties.

The main concern related to therapeutic agents for preterm birth is the potential toxicity in the developing fetus. TRX is naturally produced and ubiquitously expressed in normal human tissues or cells. From a safety standpoint, we used rhTRX for preventing preterm birth of the mouse model in this study and showed that rhTRX was effective in a prophylactic protocol as well as a therapeutic protocol against LPS. The preterm delivery rates of LPS-treated mice in prophylactic and therapeutic protocol were 94 and $68 \%$, respectively. LPS-induced preterm delivery rate between prophylactic and therapeutic protocols was not significant $(P=0.1$, Fisher's exact test).

In the human chorioamnionitis placenta, neutrophils first accumulate in the intervillous space immediately below the chorionic plate, which forms the roof of this space. The neutrophils in the roof of the intervillous space later extend upwards into the chorionic plate and reach the amnion of the fetus. At this stage, the neutrophils are purely maternal in origin in humans, being derived from maternal blood in the intervillous space (5). In placentas from rhTRX-treated mice, the administration of rhTRX appeared to prevent neutrophil activation into decidual tissue through the endothelial cells lining the blood vessels. The inhibitory effect of rhTRX on neutrophil activation appears to have an important role in attenuating the rate of preterm birth. The administration of rhTRX ameliorated severe maternal vascular dilatation and congestion in the placental labyrinth, which was the most consistent finding in LPSinduced preterm delivery model (26). Majority of the dilated 


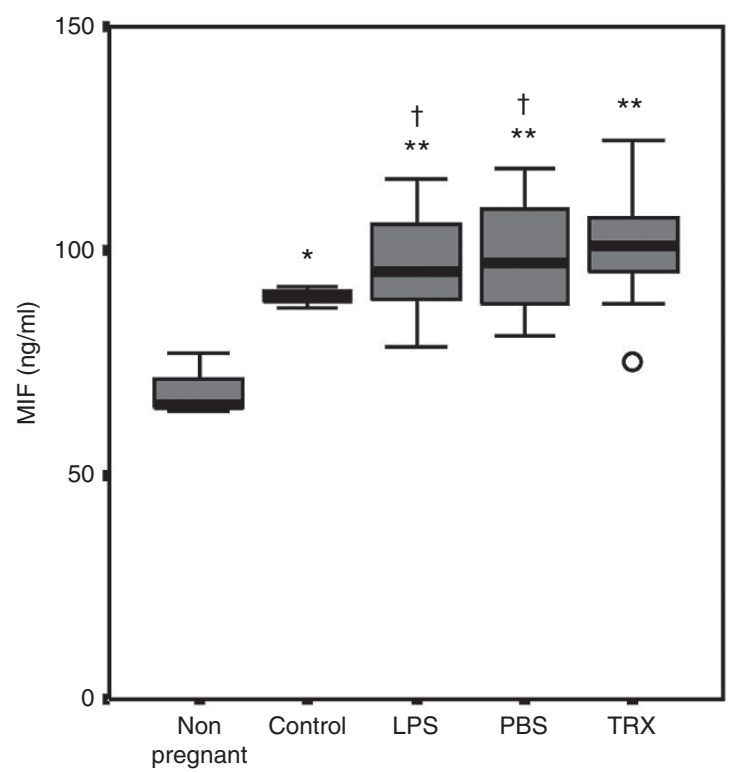

Figure 5. Migration inhibitory factor (MIF) serum level during lipopolysaccharide (LPS)-induced inflammation. Concentration of MIF was measured by ELISA in serum of nonpregnant mice (nonpregnant) and nontreated (control), LPS (LPS), LPS-PBS (PBS), and LPS-prophylactic thioredoxin-1 (TRX) (TRX) dams, $6 \mathrm{~h}$ after first LPS injection. Box-and-whisker representations indicate the median and the overall range of distribution of the measurements, the bars indicating the smallest and greatest value except for outliers. ${ }^{*} P<0.05 ; * *<0.01$ : significantly different from nonpregnant group. ${ }^{\dagger} P<0.05$ : significantly different from nontreated group.

Table 2. Effect of therapeutic TRX on a LPS-induced preterm delivery mouse model

\begin{tabular}{lcccc}
\hline & & \multicolumn{2}{c}{ Incidence $(n)$} & \\
\cline { 3 - 4 } Group & Mice $(n)$ & $\begin{array}{l}\text { Preterm } \\
\text { delivery }\end{array}$ & $\begin{array}{c}\text { Term } \\
\text { delivery }\end{array}$ & $\begin{array}{c}\text { Rate of preterm } \\
\text { delivery }(\%)\end{array}$ \\
\hline LPS & 22 & 15 & 07 & 68 \\
LPS-PBS & 34 & 23 & 11 & 68 \\
LPS-TRX & 34 & 14 & 20 & $41^{*}$ \\
\hline
\end{tabular}

Therapeutic protocol, from $1 \mathrm{~h}$ after the first LPS injection, mice received three i.V. injections of PBS containing either TRX or the vehicle, with a $3 \mathrm{~h}$ interval between injections (at 1, 4, and $7 \mathrm{~h}$ ) (Figure 1b).

The chi-square test was applied for comparison of preterm delivery rate in each group. ${ }^{*} P<0.05$ vs. LPS-PBS.

LPS, lipopolysaccharide; PBS, phosphate-buffered saline; TRX, Thioredoxin-1.

vessels were maternal, as judged from the paucity of nucleated fetal blood cells. Rounioja et al. (27) suggested that the fetal vessels in the labyrinth were severely engorged due to external compression by edematous extravascular tissue, resulting in an inappropriate increase in placental vascular resistance.

The increased production of proinflammatory mediators, such as TNF- $\alpha$ and IL-1, together with other inflammatory agents may lead to increased permeability in gestational tissues, resulting in acute congestion (26). Our data showed that rhTRX suppressed LPS-induced production of systematic TNF- $\alpha$, IFN- $\gamma$, MCP-1, and IL- 6 in sera from pregnant mice. TRX may improve maternal and fetal hemodynamic deterioration, which avoids placental congestion, in part by suppressing proinflammatory cytokines.
One of the mechanisms of suppression of LPS-induced cytokine production by TRX is that TRX directly associates with MIF and inhibits its expression and release (28). MIF has been classified as a powerful cytokine, capable of inducing TNF- $\alpha$, IL-1 $\beta$, IL-6, and IL-8, and amplifying LPS-driven cytokine responses (29). However, in the present study, we observed no difference in MIF serum levels between rhTRX-treated and untreated pregnant mice.

In conclusion, we have demonstrated that rhTRX ameliorates the rate of preterm delivery by its inhibitory effects on maternal systemic and placental inflammation. Considering the potent protective role observed in this study, rhTRX may represent a new therapeutic molecule for treatment of preterm delivery.

\section{METHODS \\ Animals}

Animal protocols were approved by the Institutional Animal Care and Use Committee of Osaka Medical Center and Research Institute for Maternal and Child Health. C3H/HeN female mice and B6D2F1 male mice were purchased from Charles River Laboratories (Yokohama, Japan). All animal experiments were performed in accordance with the guidelines of Osaka Medical Center and Research Institute for Maternal and Child Health for animal and recombinant DNA experiments. All mice were maintained under conventional housing conditions and had free access to commercial food (Oriental Yeast, Tokyo, Japan) and water.

\section{Mouse Model of Inflammation-Induced Preterm Delivery}

$\mathrm{C} 3 \mathrm{H} / \mathrm{HeN}$ female mice (9-14wk old) were pair-mated with male $\mathrm{B} 6 \mathrm{D} 2 \mathrm{~F} 1$ mice. The day on which a vaginal plug was detected was designated as gestational day 0 . All untreated pregnant mice delivered pups at gestational day 19 or 20 . Animals in the preterm delivery models were treated according to the method reported by Kaga et al. (30). On gestational day 15, two i.p. injections of LPS $(200 \mu \mathrm{g} / \mathrm{kg})$ from $E$. coli (serotype O55: B5; Sigma-Aldrich Corporation, St. Louis, MO) dissolved in $10 \mathrm{ml} / \mathrm{kg}$ of sterile saline were administered, with a 3-h interval between the injections (at 0 and $3 \mathrm{~h}$ ).

\section{rhTRX Administration}

rhTRX was supplied by Redox Bio Science (Kyoto, Japan). The quality and purity of the rhTRX used for the present study were approved by Pharmaceuticals and Medical Devices Agency in Japan, which is equivalent to the Food and Drug Administration in the United States. Its safety and kinetics were established in preclinical studies using animal models (31).

To investigate the effects of TRX on maternal systemic and placental inflammation, two protocols were designed using the LPS-induced preterm delivery model. From $1 \mathrm{~h}$ before the first LPS injection, mice received three i.v. tail vein injections of PBS $(10 \mathrm{ml} / \mathrm{kg})$, containing either rhTRX $(2 \mathrm{mg} / \mathrm{kg})$, OVA (Albumin, Egg, Nacalai tesque, Kyoto: OVA; $2 \mathrm{mg} / \mathrm{kg}$ ), or vehicle, with a 3 -h interval between injections (at $-1,2$, and $5 \mathrm{~h}$ ). The assessment of the presence or absence of delivery was performed in the early morning and the late afternoon every day throughout the experimental period. Delivery at gestational day $<19$ was defined as preterm and delivery at gestational day 19 or $>19$ was defined as full-term (prophylactic protocol, Figure 1a). From $1 \mathrm{~h}$ after the first LPS injection, mice received three i.v. injections of $10 \mathrm{ml} / \mathrm{kg}$ of PBS containing either rhTRX $(2 \mathrm{mg} / \mathrm{kg})$ or the vehicle, with a $3 \mathrm{~h}$ interval between injections (at 1, 4, and 7h) (therapeutic protocol, Figure 1b).

\section{Histochemistry and Immunohistochemistry}

Mice were euthanized by carbon dioxide inhalation $6 \mathrm{~h}$ after the first LPS injection in prophylactic protocol. Intact uteroplacental units and fetuses were harvested, fixed in $10 \%$ formalin, and embedded in paraffin (sampling protocol). Placental structures were identified on $3-\mu \mathrm{m}$ paraffin slides by hematoxylin and eosin 


\section{Articles $\quad$ Nambaet al.}

a

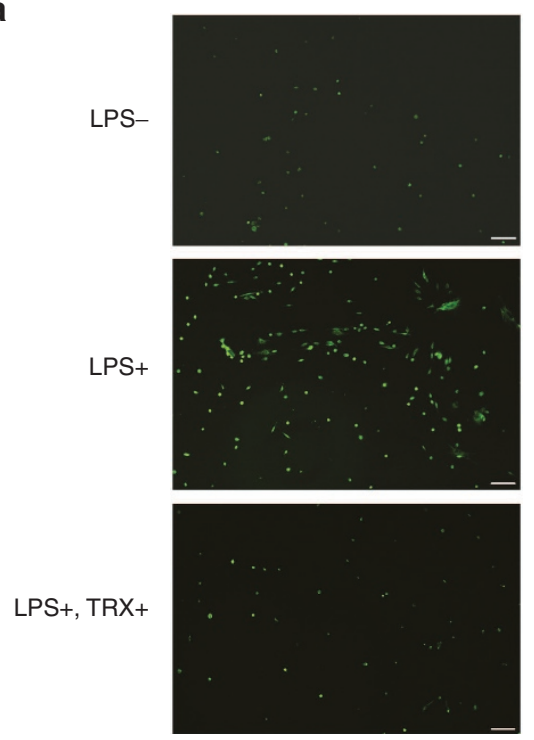

b

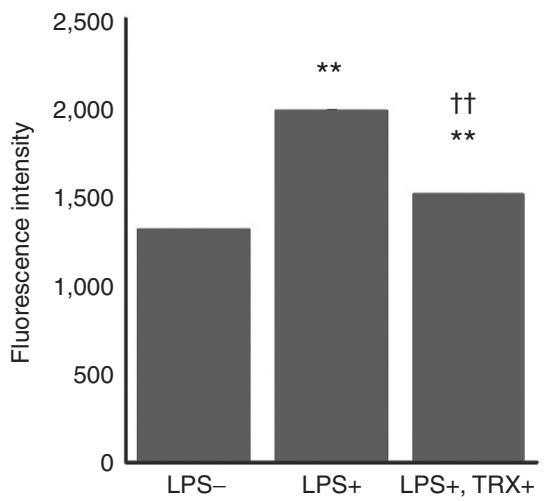

Figure 6. Reactive oxygen species (ROS)-scavenging properties of thioredoxin-1 in the mouse macrophage in vitro. (a) Representative images of ROS production assay. (b) Quantification of ROS production. Mean fluorescence units were calculated from three independent experiments of the fluorescence microscopic observations of at least 400 cells. ${ }^{* *}, p<0.001$ : significantly different from nontreated group. ${ }^{+\dagger} P<0.001$ : significantly different from lipopolysaccharide-treated group.

stain (Mayer's hematoxylin and 1\% Eosin Y Solution; Muto Pure Chemicals, Tokyo, Japan). For placental immunohistochemistry, slides were deparaffinized through three rinses in xylene (Wako Pure Chemical Industries, Osaka, Japan) and rehydrated through graded ethanol to deionized water. To detect neutrophils, a rat monoclonal antibody (NIMP-R14) directed against the neutrophil membrane (Abcam, Cambridge, UK) diluted to $1 / 500$ in PBS was used. After antigen retrieval and blocking endogenous peroxidase activity, incubation was processed overnight at $4{ }^{\circ} \mathrm{C}$. After successive rinses, immunoreactivity was detected using peroxidaselabeled affinity-purified antibody to rat immunoglobulin G (KPL Europe, Guildford, UK) or Takara POD conjugate anti-Rat for mouse tissue (Takara Bio, Otsu, Japan). Color development was performed using diaminobenzidin solution (Wako Pure Chemical Industries, Osaka, Japan), and tissue sections were counterstained with hematoxylin (Muto Pure Chemicals, Tokyo, Japan). Decidual large vessel neutrophils were then counted in each 4 mice placentas obtained from LPS treatment or prophylactic protocol treatment. Observed neutrophils were classified into two groups, inactivated neutrophils; sole and intravenously located with normal structure, and activated neutrophils; aggregated and attached to endothelial cells with deformed shape. At least 4 microscopic photographs were taken in each placenta. Total neutrophils and activated neutrophil were counted in the same magnified microscopic field (an objective lens at $\times 20)$.

\section{Cytokine Measurement in Maternal Serum}

Mice were euthanized by carbon dioxide inhalation $6 \mathrm{~h}$ after the first LPS injection in prophylactic protocol. The maternal blood samples were allowed to coagulate overnight at room temperature and subsequently centrifuged at $10,000 \times \mathrm{g}$ for $20 \mathrm{~min}$ at $4{ }^{\circ} \mathrm{C}$. The serum was carefully removed and stored at $-20{ }^{\circ} \mathrm{C}$ until used. Mouse IL-12, TNF- $\alpha$, IFN$\gamma$, MCP-1, IL-10, and IL-6 levels were determined in maternal serum using a cytometric bead array kit (BD Cytometric Bead Array Mouse Inflammation Kit; BD Biosciences, San Diego, CA) according to the manufacturer's instructions. In brief, $50 \mu \mathrm{l}$ of mouse inflammation capture bead suspension and $50 \mu \mathrm{l}$ of phycoerythrin detection reagent were added to equal amounts of the sample or standard dilution and incubated for $2 \mathrm{~h}$ at room temperature in the dark. Subsequently, samples were washed by adding $1 \mathrm{ml}$ of wash buffer and centrifuging at $200 \times \mathrm{g}$ at room temperature for $5 \mathrm{~min}$. The supernatant was discarded and $300 \mu \mathrm{l}$ of wash buffer were added. Samples were analyzed on a BD FACScan Flow Cytometer (BD Biosciences) according to the manufacturer's instructions.
The serum levels of mouse MIF were determined by the mouse MIF enzyme-linked immunosorbent assay kit (Sapporo Immuno Diagnostic Laboratory, Sapporo, Japan).

\section{ROS production assay}

ROS production assay in cultured peritoneal macrophages of C57BL/6J mice (Japan SLC, Inc., Shizuoka, Japan) were performed according to our previous report (14) with slight modification. In brief, $1 \mathrm{ml}$ of $3 \%$ sterilized fluid thioglycollate medium II (Eiken Chemical, Tokyo, Japan) was injected into the intraperitoneal cavities of 8-12-wk-old nonpregnant female mice. Three days later, the refluxed intraperitoneal fluid was collected, and macrophages were kept in the RPMI 1640 medium (Sigma) containing 10\% fetal calf serum, $100 \mathrm{U} / \mathrm{ml}$ of penicillin $\mathrm{G}$ (Sigma), and $100 \mathrm{mg} / \mathrm{ml}$ of streptomycin (Sigma). Macrophages were plated on 12 -well plate and incubated with $2 \mu \mathrm{g} / \mathrm{ml}$ of LPS (E coli O55:B5; Sigma) for $1 \mathrm{~h}$ at $37^{\circ} \mathrm{C}$ and incubated with $20 \mu \mathrm{g} / \mathrm{ml}$ of TRX for $6 \mathrm{~h}$ at $37^{\circ} \mathrm{C}$. Next, the cells were incubated for an additional $30 \mathrm{~min}$ at $37^{\circ} \mathrm{C}$ and stained with $5 \mu \mathrm{mol} / \mathrm{lCellROX}$ reagent (Life Technologies). After incubation, the cells were washed three times with PBS. ROS production was expressed in reactive fluorescence units by using a fluorescence microscope (Eclipse Ti; Nikon, Tokyo, Japan) with a filter for green fluorescent protein (86009; Chroma Technology, Bellow Falls, VT) was used, and images were acquired using DS-Qi2 (Nikon). The images were analyzed using NIS Elements software (Nikon). For the quantification of ROS production in each study groups, mean fluorescence units were calculated from three independent experiments of the fluorescence microscopic observations of at least 400 cells.

\section{Data Analysis}

Statistical analysis was performed using the software Statistical Package for the Social Sciences (SPSS) 11.0 for Windows (SPSS, Chicago, IL). The results of each experiment were expressed as the incidence and rate of preterm and term deliveries and as a box-and-whisker representation (the median, quartile, and range). The differences between each group were analyzed by Fisher's exact test (Table 1), chi-square test (Table 2), Student's $t$-test (Figure 3), Mann-Whitney $U$-test (Figures 4 and 5), or Tukey's post-hoc test with one-way ANOVA (Figure 6). The difference was considered to be statistically significant at $P<0.05$.

\section{ACKNOWLEDGMENTS}

The authors thank Y. Endo (IVR, Kyoto Univ.), T. Wakimoto, K. Nakahira (MCH), and K. Takada (BOST, Kinki Univ) for their helpful discussions and technical support. 


\section{STATEMENT OF FINANCIAL SUPPORT}

This work was supported by grants-in-aid from the Ministry of Education, Culture, Sports, Science and Technology (MEXT) (I.Y., A.K.), Japan; Development of Systems and Technology for Advanced Measurement and Analysis (Sentan), Japan Science and Technology Agency (I.Y.), Japan.

Disclosures: There are no financial ties to products in the study or potential/ perceived conflicts of interest.

\section{REFERENCES}

1. Blencowe H, Cousens S, Chou D, et al.; Born Too Soon Preterm Birth Action Group. Born too soon: the global epidemiology of 15 million preterm births. Reprod Health 2013;10 Suppl 1:S2.

2. Rubens CE, Sadovsky Y, Muglia L, Gravett MG, Lackritz E, Gravett C. Prevention of preterm birth: harnessing science to address the global epidemic. Sci Transl Med 2014;6:262sr5.

3. Kemp MW. Preterm birth, intrauterine infection, and fetal inflammation. Front Immunol 2014;5:574.

4. Romero R, Espinoza J, Gonçalves LF, Kusanovic JP, Friel L, Hassan S. The role of inflammation and infection in preterm birth. Semin Reprod Med 2007:25:21-39.

5. Namba F, Hasegawa T, Nakayama M, et al. Placental features of chorioamnionitis colonized with Ureaplasma species in preterm delivery. Pediatr Res 2010;67:166-72.

6. Uchida K, Nakahira K, Mimura K, et al. Effects of Ureaplasma parvum lipoprotein multiple-banded antigen on pregnancy outcome in mice. J Reprod Immunol 2013;100:118-27.

7. Cha J, Bartos A, Egashira M, et al. Combinatory approaches prevent preterm birth profoundly exacerbated by gene-environment interactions. J Clin Invest 2013;123:4063-75.

8. Tagaya Y, Maeda Y, Mitsui A, et al. ATL-derived factor (ADF), an IL-2 receptor/Tac inducer homologous to thioredoxin; possible involvement of dithiol-reduction in the IL-2 receptor induction. EMBO J 1989;8:757-64.

9. Yoshihara E, Masaki S, Matsuo Y, Chen Z, Tian H, Yodoi J. Thioredoxin/ Txnip: redoxisome, as a redox switch for the pathogenesis of diseases. Front Immunol 2014;4:514.

10. Ahsan MK, Lekli I, Ray D, Yodoi J, Das DK. Redox regulation of cell survival by the thioredoxin superfamily: an implication of redox gene therapy in the heart. Antioxid Redox Signal 2009;11:2741-58.

11. Nakamura H, Herzenberg LA, Bai J, et al. Circulating thioredoxin suppresses lipopolysaccharide-induced neutrophil chemotaxis. Proc Natl Acad Sci USA 2001;98:15143-8.

12. Okuyama $H$, Nakamura $H$, Shimahara $Y$, et al. Overexpression of thioredoxin prevents acute hepatitis caused by thioacetamide or lipopolysaccharide in mice. Hepatology 2003;37:1015-25.

13. Hoshino T, Nakamura H, Okamoto M, et al. Redox-active protein thioredoxin prevents proinflammatory cytokine- or bleomycin-induced lung injury. Am J Respir Crit Care Med 2003;168:1075-83.

14. Wakimoto T, Uchida K, Mimura K, et al. Hydroxylated fullerene: a potential antiinflammatory and antioxidant agent for preventing mouse preterm birth. Am J Obstet Gynecol 2015;213:708.e1-9.

15. Higby K, Xenakis EM, Pauerstein CJ. Do tocolytic agents stop preterm labor? A critical and comprehensive review of efficacy and safety. Am J Obstet Gynecol 1993;168:1247-56; discussion 1256-9.
16. Dodd JM, Jones L, Flenady V, Cincotta R, Crowther CA. Prenatal administration of progesterone for preventing preterm birth in women considered to be at risk of preterm birth. Cochrane Database Syst Rev 2013;7:CD004947.

17. Blencowe H, Cousens S, Oestergaard MZ, et al. National, regional, and worldwide estimates of preterm birth rates in the year 2010 with time trends since 1990 for selected countries: a systematic analysis and implications. Lancet 2012;379:2162-72.

18. Chang HH, Larson J, Blencowe H, et al.; Born Too Soon preterm prevention analysis group. Preventing preterm births: analysis of trends and potential reductions with interventions in 39 countries with very high human development index. Lancet 2013;381:223-34.

19. Schmitz T, Souil E, Hervé R, et al. PDE4 inhibition prevents preterm delivery induced by an intrauterine inflammation. J Immunol 2007;178: 1115-21.

20. Pirianov G, Waddington SN, Lindström TM, Terzidou V, Mehmet H, Bennett PR. The cyclopentenone 15-deoxy-delta 12,14-prostaglandin J(2) delays lipopolysaccharide-induced preterm delivery and reduces mortality in the newborn mouse. Endocrinology 2009;150:699-706.

21. Holmgren C, Esplin MS, Hamblin S, Molenda M, Simonsen S, Silver R. Evaluation of the use of anti-TNF-alpha in an LPS-induced murine model. J Reprod Immunol 2008;78:134-9.

22. Koscica KL, Ananth CV, Placido J, Reznik SE. The effect of a matrix metalloproteinase inhibitor on inflammation-mediated preterm delivery. Am J Obstet Gynecol 2007;196:551.e1-3.

23. Zhao M, Chen YH, Dong XT, et al. Folic acid protects against lipopolysaccharide-induced preterm delivery and intrauterine growth restriction through its anti-inflammatory effect in mice. PLoS One 2013;8: e82713.

24. Hadi T, Bardou M, Mace G, et al. Glutathione prevents preterm parturition and fetal death by targeting macrophage-induced reactive oxygen species production in the myometrium. FASEB J 2015;29:2653-66.

25. Lee JW, Davis JM. Future applications of antioxidants in premature infants. Curr Opin Pediatr 2011;23:161-6.

26. Xu DX, Wang H, Zhao L, Ning H, Chen YH, Zhang C. Effects of low-dose lipopolysaccharide (LPS) pretreatment on LPS-induced intra-uterine fetal death and preterm labor. Toxicology 2007;234:167-75.

27. Rounioja S, Räsänen J, Ojaniemi M, Glumoff V, Autio-Harmainen H, Hallman M. Mechanism of acute fetal cardiovascular depression after maternal inflammatory challenge in mouse. Am J Pathol 2005;166:1585-92.

28. Son A, Kato N, Horibe T, et al. Direct association of thioredoxin-1 (TRX) with macrophage migration inhibitory factor (MIF): regulatory role of TRX on MIF internalization and signaling. Antioxid Redox Signal 2009;11:2595-605.

29. Kudrin A, Ray D. Cunning factor: macrophage migration inhibitory factor as a redox-regulated target. Immunol Cell Biol 2008;86:232-8.

30. Kaga N, Katsuki Y, Obata M, Shibutani Y. Repeated administration of low-dose lipopolysaccharide induces preterm delivery in mice: a model for human preterm parturition and for assessment of the therapeutic ability of drugs against preterm delivery. Am J Obstet Gynecol 1996;174: 754-9.

31. Nakamura H, Hoshino Y, Okuyama H, Matsuo Y, Yodoi J. Thioredoxin 1 delivery as new therapeutics. Adv Drug Deliv Rev 2009;61:303-9. 